\title{
MedienPädagogik
}

Zeitschrift für Theorie und Praxis der Medienbildung

Themenheft Nr. 40: CoViD-19 und die digitale Hochschulbildung. Irritationen, Einsichten und Programmatiken

Herausgegeben von Markus Deimann, Marios Karapanos und Klaus Rummler

\section{Digitales Lernen während der Covid-19- Pandemie aus Sicht von Studierenden der Erziehungswissenschaften}

\author{
Handlungsempfehlungen für die Digitalisierung von Hochschullehre
}

Miriam Mulders und Sophia Krah

\begin{abstract}
Zusammenfassung
Der vorliegende Beitrag untersucht die Bedingungen von Lehren und Lernen während der Covid-19-Pandemie. Verantwortliche der Hochschullehre und Studierende waren im Sommersemester 2020 gezwungen, auf digitale Formate des Lehrens bzw. Lernens zurückzugreifen. Im Rahmen der vorliegenden Studie wird auf Grundlage einer Fragebogenstudie mit Bachelorstudierenden des Fachs Erziehungswissenschaften zunächst die derzeitige Situation und das Meinungsbild der Studierenden differenziert erfasst und dargestellt. Anschliessend werden Faktoren herausgearbeitet, die aus Studierendenperspektive zentral für den erfolgreichen Einsatz von digitalen Formaten in der Hochschullehre sind. Aufbauend darauf werden Unterstützungsbedarfe identifiziert und Handlungsempfehlungen für die Gestaltung von digitalem Lehren und Lernen formuliert. Die Untersuchungsergebnisse weisen darauf hin, dass das Sommersemester 2020 eine Herausforderung für alle Beteiligten gewesen ist. Besonders technologische Hürden erschwerten das Studieren. Auch didaktische und organisatorische Gestaltungsaspekte digitaler Lehr- und Lernkonzepte bestimmten das Studierverhalten. Die Untersuchung liefert wichtige Hinweise hinsichtlich digital (bzw. digital angereicherter) Lehre. Diese sind wegweisend, um die Lehre in der gegenwärtigen Situation erfolgreich durchzuführen und um die Qualität der Lehre und des Lernens im Hochschulbereich zu sichern.
\end{abstract}

Digital Learning during the Covid-19 pandemic from the perspective of educational science students. Recommendations for the digitalization of teaching in higher education

\begin{abstract}
The following paper examines the conditions of teaching and learning of undergraduate students during the Covid-19 pandemic. In the spring semester of 2020, those responsible for university teaching and their students have been forced to use digital formats for teaching and learning. A questionnaire study with educational science students provides
\end{abstract}


data on the current situation and the students' opinions. Afterwards, factors are identified which, from a student perspective, are central to the successful use of digital formats in university teaching. Subsequently, it is determined which support measures are needed and recommendations for the design of digital teaching and learning are defined. The results of the study indicate that the spring semester 2020 has been a challenge for all participants. Due to technological barriers, studying has been more difficult. Didactic and organisational design aspects of digital teaching and learning concepts also influenced study behaviour. The conducted research delivers relevant findings regarding digital (or digitally enriched) teaching which are important to successfully provide teaching in the current situation and to ensure the quality of teaching and learning in the higher education sector.

\section{Digitales Lehren und Lernen an Hochschulen}

E-Learning wird definiert als die Bereitstellung und Nutzung von Lehr- und Lernmaterial mithilfe elektronischer Medien und ist Sammelbegriff für sämtliche Formen mediengestützten Lernens, welche multimediale und kommunikative Technologien integrieren (Kerres 2018). Es gilt als zentraler Bestandteil und wesentlicher Schlüssel für innovative Hochschullehre (Persike und Friedrich 2016; Schünemann und Budde 2018; Metzner et al. 2019). Daher setzen Hochschulen im Rahmen der Aus- und Weiterbildung vermehrt auf digitale Formate des Lehrens und Lernens. Digitale Lernangebote wurden in den vergangenen Jahren vielfach an deutschen Hochschulen entwickelt, erprobt und eingesetzt. Von politischer Seite wurde versucht, das Wachstum von digitalem Lernen durch eine Reihe von Förderprogrammen voranzutreiben. Allein den Hochschulen standen in den letzten Jahrzehnten mehrere hundert Millionen Euro Fördergelder für deren E-Learning-Projekte zur Verfügung (Bremer et al. 2010). Vorteile der Nutzung neuartiger Bildungstechnologien in der Hochschullehre werden vor allem in der Erschliessung neuer Studentengruppen durch erhöhte studentische zeitliche und räumliche Flexibilität, in finanziellen Einsparungen, in der Steigerung internationaler Wettbewerbsfähigkeit und in der qualitativen Verbesserung von Hochschullehre gesehen (Euler und Seufert 2003; Faber 2011). Es muss dennoch konstatiert werden, dass bis heute nicht von einer flächendeckenden Nutzung mediengestützter Lehre im Hochschulalltag gesprochen werden kann (Persike und Friedrich 2016; Handke 2020). Der Dissemination von E-Learning in der Hochschullehre stehen weiterhin Herausforderungen, unter anderem in den Bereichen technologische Ausstattung, Akzeptanz und Innovationsbereitschaft, Rechtsmanagement und curriculare Integration, gegenüber (Kleimann und Wannemacher 2005; Euler und Seufert 2005). 
Für eine erfolgreiche Integration von mediengestützter Hochschullehre können nach Kerres (2018) vier Aktionsfelder definiert werden (siehe Abbildung 1). Die Aktivitäten in den Bereichen Infrastruktur, didaktische Reform, Entwicklung und Medien stehen in Abhängigkeit zueinander und sollen aufeinander abgestimmt sowie in etwa gleichgewichtet stattfinden. Zur Infrastruktur zählt die technische Ausstattung der Hochschule sowie die mit Wartung, Sicherung und Support verbundenen Dienstleistungen. Die didaktische Reform bezieht sich auf anzupassende und neu zu entwickelnde Lehr- und Lerninhalte, Konzepte sowie Methoden. Aktivitäten im Bereich Entwicklung adressieren personelle und organisationale Rahmenbedingungen. Das Aktionsfeld Medien umfasst Massnahmen zur Produktion und Distribution medialer Inhalte.

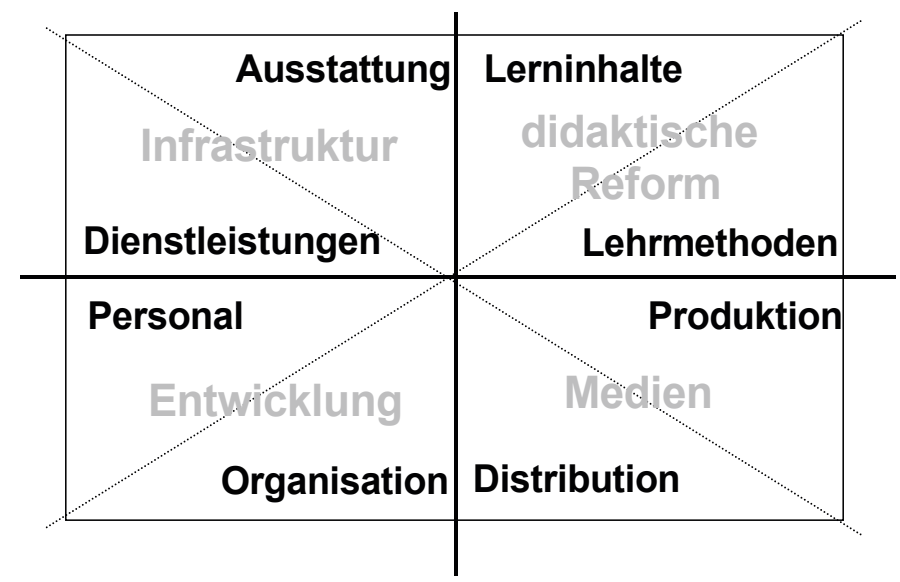

Abb. 1.: Viereck mediendidaktischer Innovation (Kerres 2018, 503).

Auch Euler und Seufert (2005) identifizieren Kriterien für eine erfolgreiche Implementierung von E-Learning an Hochschulen, darunter Ökonomie, Technologie, Organisation, Kultur und Didaktik. Ökonomische Aspekte betreffen Kosten-Nutzen-Kalkulationen, die mit der Bereitstellung der technischen Infrastruktur sowie Ressourcen zur Umsetzung und Förderung von E-Learning einhergehen. Die technologische Dimension umfasst die Funktionalität und Praktikabilität des Angebots, die massgeblich Akzeptanz und Annahmebereitschaft der Nutzenden beeinflussen. Darüber hinaus müssen organisationale Rahmenbedingungen geschaffen werden, welche einen flexiblen und effizienten Einsatz von E-Learning ermöglichen. Kulturelle Aspekte berücksichtigen Innovationsbereitschaft und Selbstorganisation aller Beteiligten. Die mediendidaktische Aufbereitung beinhaltet den erfolgreichen Transfer analoger Inhalte und Methoden in digitale bzw. digital angereicherte Konzepte.

Bedingt durch die Covid-19-Pandemie waren sämtliche Bildungsträger, darunter auch Hochschulen, gezwungen, ad hoc auf digitales Lehren und Lernen umzustellen (auch Emergency Remote Teaching, dt.: Notfall-Fern-Lehre). Bisherige Lehr- und 
Lernformate mussten kurzfristig ausschliesslich online umgesetzt werden, was viele Hochschulen, Lehrende sowie Lernende vor grössere oder kleinere Hürden stellte (Kerres 2020). Statt einer gut durchdachten und geplanten digitalen Transformation wurden sowohl Studierende als auch Lehrende innerhalb einer sehr kurzen Zeitspanne mit vielen Neuerungen und Anforderungen konfrontiert. Ein Ziel dieser Untersuchung ist es daher, ein Bild von Lehre und Lernen während des durch die Pandemie geprägten Sommersemesters 2020 aus Sicht von Bachelorstudierenden der Disziplin Erziehungswissenschaften einer deutschen Hochschule zu skizzieren und dabei Faktoren zu identifizieren, die das Studieren modifizieren. So sollen vor allem aktuell relevante Unterstützungsbedarfe und Handlungsempfehlungen für die Gestaltung von Hochschullehre abgeleitet werden. Es gilt daher, aufbauend auf den Ausführungen von Kerres (2018) sowie Euler und Seufert (2005), relevante Kriterien für eine erfolgreiche und nachhaltige Verankerung von digitalem Lehren und Lernen in der Hochschule aufzudecken. Es interessiert, inwiefern Studierende digitale Lehr- und Lernformate im Sommersemester 2020 annahmen und nutzten und welche Faktoren darauf einen Einfluss nahmen. Erfolg oder Misserfolg einer digitalen Methode hängt von dessen Akzeptanz, sowohl auf Studierenden- als auch auf Dozierendenseite ab. Akzeptanz wird dabei verstanden als «ein in Theorie und Praxis regelmässig genanntes, komplexes und nicht direkt beobachtbares psychologisches Schlüsselkonstrukt» (Rüggeberg 2009, 7). Akzeptanz wird unterschieden in latente Einstellungsakzeptanz und beobachtbare Verhaltensakzeptanz (Lehmann 2010). Kreidl (2011) benennt in diesem Zusammenhang vier zentrale Faktoren, welche die Akzeptanz der Studierenden, d. h. Annahmebereitschaft und Nutzungsverhalten von digitalen Medien, modifizieren. Diese werden im Folgenden dargestellt.

\subsection{Sozioökonomische Situation der Studierenden}

Sozioökonomische Merkmale von Studierenden, darunter Berufstätigkeit, Lebenssituation, Entfernung zum Studienort sowie die Vorbildung (z. B. durch ein vorheriges Studium oder eine Ausbildung) beeinflussen das Studierverhalten und auch inwiefern digitale Lehr- und Lernformate von der Studierendenschaft angenommen und genutzt werden (Lojewski und Schäfer 2017). Wer bereits im familiären, beruflichen oder sozialen Umfeld viel mit digitalen Werkzeugen arbeitet, dem fällt der Umgang auch im universitären Kontext leicht. Reaktanz und Überforderung bleiben aus. Wer jedoch wenig technisch affin ist, steht vor grösseren Hürden (Kreidl 2011). Es ist davon auszugehen, dass aufgrund unterschiedlicher Ausgangsvoraussetzungen der Studierenden (z. B. Zugang zu stabiler Internetverbindung, technische Ausstattung) Akzeptanz und Nutzen von digitalen Lehr- und Lernangeboten interindividuell unterschiedlich ausgeprägt sind (Lojewski und Schäfer 2017). Lehrende müssen diese Heterogenität unter Studierenden bei der Planung von E-Learning berücksichtigen 
(Kreidl 2011). Dies gilt es auch im Rahmen der Covid-19-Pandemie zu beachten. Die kurzfristige Umstrukturierung der Hochschullehre führte zu der Notwendigkeit für Studierende, ausserhalb universitärer Räumlichkeiten einen Lernort zu schaffen, der über die nötige technische Ausstattung (z. B. Laptop) und eine stabile Internetverbindung verfügt sowie ein lernfreundliches Arbeitsklima bietet.

\subsection{Didaktische Gestaltung}

Bei der Qualitätssicherung von digitalen und analogen Lernangeboten ist die didaktische Gestaltung ein zentraler Baustein (Kergel und Heidkamp-Kergel 2020). Kreidl (2011) identifiziert für die didaktische Konzeption zwei entscheidende Faktoren: «Das Anknüpfen an die Vorkenntnisse der Studierenden sowie das Bereitstellen von thematischen Strukturen» (ebd., 32). Demnach ist es von elementarer Bedeutung, den Studierenden Aufgaben zu geben, die ihrem bisherigen Wissensstand entsprechen und zudem sinnvoll strukturiert sind. Eine grosse Herausforderung besteht hierbei darin, auf der einen Seite eine Struktur vorzugeben und auf der anderen Seite Individualisierung nach Wissensstand oder Lernbedarf zu ermöglichen (Kerres 2018). Auch Ehlers (2011) beschreibt Individualisierung und Bedarfsorientierung als Motive für das digitale Lernen. Für Kreidl (2011) beinhaltet die didaktische Gestaltung die Aspekte Verständlichkeit der Inhalte, geeignete Problemstellungen und Lernanlässe, Rückmeldungen über die Qualität der Verarbeitung sowie Kommunikations- und Kooperationsanlässe.

\subsection{Organisatorische Gestaltung}

Bei der Konzeption der Lernumgebung und des Lernprozesses gilt es, verschiedene organisatorische Faktoren zu berücksichtigen. Nach Kreidl (2011) bringt das Lernen mit digitalen Medien viele neue Herausforderungen mit sich, sodass seitens der Hochschulen unterstützende Massnahmen auf inhaltlicher und technischer Ebene etabliert werden sollten. Darüber hinaus fordern Studierende Transparenz hinsichtlich abzulegender Prüfungsleistungen, strukturierte Abläufe und eine klare Kommunikation. Zur organisatorischen Gestaltung wird des Weiteren die technische Umsetzung des Angebots gezählt. Nutzerfreundlichkeit und Funktionalität von digitalen Lernumgebungen prägen das Studierverhalten (Persike und Friedrich 2016). Auch Ehlers (2011) betont, "die Lernplattform - im Sinne technischer Funktionalitäten sei ein Faktor, der die Motivation» (ebd., 180) beeinflussen kann. 


\subsection{Studien- und Lernmerkmale}

Ein weiterer Punkt, der bei der Analyse von Nutzungsverhalten und Akzeptanz zu berücksichtigten ist, sind Studien- und Lernmerkmale. Ehlers (2011) schreibt hierzu, dass das Potenzial digitaler Angebote nur dann vollkommen genutzt werden kann, wenn die Lernenden «eine hohe (...) Lernkompetenz» (ebd., 105) besitzen. Hier hebt er besonders eine selbstständige Arbeitsweise hervor. Kreidl (2011) schliesst daran an und postuliert einen möglichen Zusammenhang zwischen dem bisherigen Studienerfolg und Akzeptanz, Nutzung und Erfolg eines E-Learning Angebots. Zudem beschreibt er, dass die bisherige Studiendauer, insbesondere im Hinblick auf bereits erworbene fachliche und methodische Kompetenzen, einen Faktor ausmacht. Unabhängig vom Studium, sind auch Vorkenntnisse mit E-Learning wichtig zu betrachten. «Besonders interessant [bei geringen Vorkenntnissen] ist in diesem Zusammenhang, dass sowohl ein positiver Effekt (hohe Akzeptanz durch die Neuartigkeit und Abwechslung) als auch ein negativer Effekt (niedrige Akzeptanz durch mangelnde Vertrautheit mit dem System) denkbar ist» (Kreidl 2011, 46).

\section{Forschungsfragen und Hypothesen}

In Kreidls Untersuchung (2011) liessen sich signifikante Zusammenhänge zwischen organisatorischen und didaktischen Gestaltungsaspekten und der Akzeptanz von digitalen Lehr- und Lernformaten seitens der Studierendenschaft nachweisen, wobei die didaktische Aufbereitung weitaus relevanter zu sein scheint als organisatorische Einflüsse. Sozioökonomische Faktoren und Studien- und Lernmerkmale korrelierten nicht signifikant mit Akzeptanzparametern. Nichtsdestotrotz erscheint eine Betrachtung aller vier von Kreidl (2011) postulierten Faktoren - insbesondere auch vor dem Hintergrund der Auswirkungen der Covid-19-Pandemie - als sinnvoll und erkenntnisbringend.

Im Rahmen dieser Publikation sollen aus der Perspektive von Bachelorstudierenden der Erziehungswissenschaften zentrale Bedingungen identifiziert werden, die für das Gelingen von reinen E-Learning-Formaten notwendig sind. Die Kenntnis solcher Gelingensbedingungen ist derzeit von enormer Bedeutung, um Studieren, das ausschliesslich online stattfinden kann, allen Studierenden zu ermöglichen und qualitativ hochwertig zu gestalten. Aber auch über die Covid-19-Pandemie hinaus, haben die Erkenntnisse Hinweischarakter, wie erfolgreiche digitale Lehre im Hochschulsektor gestaltet sein kann. Wannemacher und Kollegen führten 2016 eine Studie zu digitaler Lehre an deutschen Hochschulen durch. Die Erhebung zeigte, dass digitale Lehr- und Lernformen an einem Grossteil der Hochschulen vorhanden sind, diese jedoch in Abhängigkeit von Hochschultyp, -trägerschaft und -grösse erheblich variieren können. Auch die Wahrnehmung von Herausforderungen im Bereich der digitalisierten Lehre differierte stark. Es wurden Herausforderungen u.a. in knappen 
Personalressourcen und Akzeptanzproblemen bei Lehrenden und Studierenden verortet. Herausforderungen resultieren häufig in niedrigen Nutzungszahlen. Es ist daher bedeutsam, die Hintergründe fehlender studentischer Akzeptanz und niedriger Nutzungsraten von digitalen Lehr- und Lernangeboten im Hochschulsektor zu erforschen. Auch das BMBF geförderte You(r) Study Forschungsprojekt (u.a. Becker, Riplinger und Schiefner-Rohs 2020) zur Rolle digitaler Medien bei der Gestaltung von Lehr-Lernarrangements erforscht Gelingensbedingungen digitaler Lernarrangements seitens der Studierendenschaft.

Zusammenfassend lässt sich festhalten, dass der Einsatz von E-Learning als Innovation im Bildungsbereich mit diversen Hürden konfrontiert wird. Die Zielsetzung dieses Papiers besteht daher erstens in der deskriptiven Beschreibung der Lehr- und Lernsituation im Sommersemester 2020, in welchem im Zuge der Covid-19-Pandemie oftmals eine abrupte Digitalisierung der Hochschullehre stattgefunden hat. Es soll der Frage nachgegangen werden, ob die Pandemie bereits zum jetzigen Zeitpunkt Hochschullehre zur Veränderung angestossen hat. Zweitens versucht das Papier erste Kriterien zu identifizieren, die Annahmebereitschaft, Akzeptanz und Nutzung von E-Learning Angeboten im Hochschulbereich modifizieren. Dahinter steht die Absicht, Kenntnisse über Gelingensbedingungen von digitalen Lehr- und Lernformaten aus der Perspektive der Studierenden zu gewinnen.

Daraus hervorgehend werden folgende Forschungsfragen innerhalb der vorliegenden Untersuchung näher beleuchtet:

- Wie haben die Studierenden das von der Covid-19-Pandemie geprägte Sommersemester 2020 wahrgenommen?

- Was sind aus Studierendenperspektive Gelingensbedingungen von digitalem Lernen?

- Welche initialen Handlungsempfehlungen für Lehrende lassen sich ableiten?

Aus den Fragestellungen und der Literatur ergeben sich folgende Hypothesen:

- Im Sommersemester 2020 wurde mehr Zeit für die Universität aufgewendet als die Semester davor.

- Studierende haben das Sommersemester 2020 als besonders herausfordernd empfunden.

- Innerhalb des Sommersemesters 2020 haben sich bestimmte digitale Werkzeug etabliert.

- Studierende bezeichnen sich am Ende des Sommersemester 2020 eher als Digital Natives als zu Semesterbeginn.

- Studierende bevorzugen asynchrone E-Learning Formate gegenüber synchronen.

- Studierende lernen lieber zur bestimmten von Lehrenden festgelegten Zeiten als zu individuellen Zeitpunkten, die selbst festgelegt werden.

- Studierende lernen lieber allein als in Gruppen. 
- Studierende wünschen sich eine Mischung aus digitalen und analogen Elementen in der Lehre.

- Die sozioökonomische Situation von Studierenden hängt mit Akzeptanz und Nutzungsverhalten von E-Learning Formaten zusammen.

- Je besser die organisatorische Gestaltung eines E-Learning Angebots, desto höher die Akzeptanz und Nutzung durch Studierende.

- Je besser die didaktische Gestaltung eines E-Learning Angebots, desto höher die Akzeptanz und Nutzung durch Studierende.

- Studien- und Lernmerkmale hängen mit Akzeptanz und Nutzungsverhalten von E-Learning Formaten zusammen.

\section{Forschungsmethodik}

Um die Hypothesen der Untersuchung zu überprüfen, wurden quantitative Methoden genutzt. Zu zwei Messzeitpunkten (MZP) wurden Studierende aus zwei Parallelseminaren des Bachelorstudiengangs Erziehungswissenschaften der Universität Duisburg-Essen gebeten, einen Online-Fragebogen auszufüllen. Die Fragebögen bestanden aus geschlossenen und offenen Fragen mit unterschiedlichen Antwortformaten. Neben deskriptiven Merkmalen, wurde zu MZP 1 das Nutzungsverhalten digitaler Werkzeuge und Plattformen untersucht. Zu MZP 2 wurden die sozioökonomische Situation, Einstellungen zu digitalen Werkzeugen und Plattformen sowie zur Lehr- und Lernsituation während der Covid-19-Pandemie und in Zukunft erfragt. Darüber hinaus wurde der Fragebogen von Kreidl (2011) adaptiert, um zu untersuchen, inwieweit Zusammenhänge zwischen den Aspekten sozioökonomische Situation der Studierenden, didaktische Gestaltung, organisatorische Gestaltung sowie Studienund Lernmerkmale und studentischem Akzeptanz- und Nutzungsverhalten von ELearning Angeboten bestehen. Diesem Fragebogen liegt als Antwortformat eine fünfstufige Likert-Skala von stimme gar nicht zu bis stimme vollkommen zu (z. B. «Die in diesem Semester eingesetzten E-Learning Angebote finde ich insgesamt nützlich.»). Die Items des Fragebogens werden in Skalen zusammengefasst, um die intendierten Konstrukte (z. B. didaktische Gestaltung) zu erfassen. Die Stichprobengrösse variiert zwischen den MZP und reicht von 39 (MZP 1) bis 19 (MZP 2). Die Auswertung der quantitativen Daten erfolgte mit Hilfe des Statistikprogramms SPSS für Macintosh (IBM SPSS Statistics 22.0).

\section{Ergebnisse}

Im Folgenden sollen zuerst deskriptive Kennwerte der Stichprobe beschrieben werden. Darauffolgend werden die subjektiven Einstellungen der Studierenden zu digitaler Lehre während des von Covid-19 geprägten Sommersemesters 2020 dargeboten. 
Im letzten Schritt sollen die angenommenen Zusammenhänge zwischen Akzeptanz und Nutzen von E-Learning Angeboten und diversen Kontextfaktoren thematisiert werden.

\subsection{Deskriptive Daten der Stichprobe}

Das durchschnittliche Alter der Stichprobe liegt bei 22 Jahren. Entsprechend der üblichen Geschlechterverteilung in diesem Studiengang (Klinger 2015) sind $92 \%$ der untersuchten Studierenden weiblich, $8 \%$ männlich. Die durchschnittliche Anzahl bereits studierter Semester liegt bei vier. $100 \%$ der Befragten gaben an, in Vollzeit zu studieren. Keiner der Studierenden berichtet, bereits Kinder zu haben. $26 \%$ leben mit einem Partner oder einer Partnerin in einer Gemeinschaft zusammen. Bei $90 \%$ ist die Muttersprache deutsch. Der Weg zur Hochschule beträgt bei $5 \%$ unter zehn Minuten, bei $21 \%$ zwischen elf und 29 Minuten, bei $42 \%$ zwischen 30 und 59 Minuten und bei weiteren $31 \%$ zwischen ein und zwei Stunden. $68 \%$ bejahten die Frage, eine stabile Internetverbindung an ihrem Wohnort zu haben, $32 \%$ gaben an, dass dies nur teilweise der Fall sei. 95 \% bestätigten, über einen Laptop an ihrem Wohnort verfügen zu können, bei $5 \%$ ist dies nur teilweise der Fall. Auf die Frage, welche mobilen Endgeräte besessen werden, antworteten $92 \%$ Laptop, 97 \% Smartphone und $46 \%$ Tablet.

\subsection{Deskriptive Beschreibung der Lehr- und Lernsituation während der Covid-19- Pandemie}

Über mehrere offene und geschlossene Fragenformate wurden studentische Eindrücke und Einstellungen zur Lehr- und Lernsituation während der Covid-19-Pandemie im Sommersemester 2020 erfasst. Aufgrund der kleinen Stichprobe liessen sich kaum inferenzstatistische Verfahren berechnen, um die oberhalb benannten und a priori festgelegten Hypothesen angemessen zu prüfen. Daher beschränkt sich der folgende Ergebnisteil der vorliegenden Untersuchung auf die Präsentation von deskriptiven Häufigkeiten und Zitaten aus Freitextantworten der Studierenden. Die Erkenntnisse sind daher mit Vorsicht zu betrachten und haben vielmehr einen Hinweischarakter für Forschung und Praxis.

Viele der Studierenden berichten von einer stärkeren Selbststeuerung ihrer Lernprozesse (z. B. «flexible Zeiteinteilung») als die Semester zuvor. Konträr benennen die Studierenden aber auch, dass sie durch viele externe Strukturen gelenkt worden seien. So habe es in diesem Semester viele «kleinschrittige» Lernaufgaben gegeben, die meist wöchentlich erledigt werden mussten. Häufig habe die Lehre asynchron stattgefunden. «Viele Texte [wurden] hochgeladen zum Lesen und Bearbeiten.» 
In der ersten Hypothese wurde postuliert, dass im Sommersemester 2020 mehr Zeit für die Universität aufgewendet wurde als die Semester zuvor. Wie oben bereits erläutert, war eine inferenzstatistische Hypothesenprüfung nicht möglich. Daher seien für letztere sowie sämtliche folgende Hypothesen Häufigkeiten dargeboten. $53 \%$ gaben an, mehr Zeit aufgewendet zu haben als sonst, $32 \%$ verneinten dies und weitere $15 \%$ konnten dies nicht einschätzen. Studierende berichten «Jeder [Dozent bzw. jede Dozentin] hat für seinen Kurs viel mehr Zeit beansprucht.» oder «[Es war] viel mehr Arbeitserwartung an Studierende als im Normalzustand.».

Wie angenommen, hat ein Grossteil der Studierenden das benannte Semester als Herausforderung empfunden. So war für $5 \%$ das Semester kaum, für $42 \%$ teilweise, für $32 \%$ überwiegend und für $21 \%$ vollkommen herausfordernd.

Zudem wurde moniert, dass «viel weniger Austausch als sonst» stattgefunden habe. Nach Studierendenmeinung hat die virtuelle Kommunikation nur für $5 \%$ vollkommen, für $16 \%$ überwiegend, für $26 \%$ teilweise, für $37 \%$ kaum und für $16 \%$ sogar gar nicht so gut funktioniert wie sonst in Präsenz. Der Wunsch nach Lehre in Präsenz wird in den Freitextantworten häufig mit dem Wunsch nach fachlicher und zwischenmenschlicher Kommunikation verknüpft.

Ergänzend muss erwähnt werden, dass das Engagement der Lehrenden von den Studierenden als sehr heterogen empfunden wurde («Die Dozenten geben sich unterschiedlich viel Mühe und nutzen unterschiedlich viele digitale Möglichkeiten», "[Manche hätten] sicherlich eine Weiterbildung in Bezug auf die Digitalisierung ihrer Lehre und vor allem auf die Aktivierung der Studierenden gut gebraucht.»).

Darüber hinaus haben sich neue digitale Werkzeuge unter den Studierenden im Sommersemester 2020 etabliert. Dazu zählen insbesondere Videokonferenztools. Benannt wurden Zoom, BigBlueButton, Jitsi und Microsoft Teams, wobei Zoom am meisten zu überzeugt haben scheint («Es gibt viele Möglichkeiten miteinander zu agieren, in Kleingruppen zu arbeiten und alle zu sehen. Es fühlt sich fast an, als sässe man gemeinsam in einem Raum.»). Für das kollaborative, gemeinsame Erarbeiten von Inhalten wurde Padlet als neu erlerntes Werkzeug genannt, «weil einfach die Möglichkeit besteht, ganz viele Eindrücke zu sammeln und zu sortieren». Insgesamt sind $64 \%$ Prozent der Studierenden vollkommen oder überwiegend der Ansicht, dass digitale Werkzeuge sie beim Lernen unterstützen (37\% teilweise, $0 \%$ kaum oder gar nicht).

Es bestand die Annahme, dass Studierende im Verlauf des Sommersemesters 2020 digital affiner werden. So sollten sich die Studierenden zu beiden MZP auf einer Skala von 0 (niedrige Ausprägung) bis 100 (hohe Ausprägung) einordnen, wie sehr sie sich selbst als Digital Native, eine Person, die von Kindheit an mit Informationstechnologien und dem Internet aufgewachsen ist und eine Welt ohne digitale Medien nicht kennt (Heiden et al. 2013), wahrnehmen. Zu MZP 1 lag der Durchschnittswert bei 63.5, zu MZP 2 bei 62.9. Auch hinsichtlich der Nutzung des Lern-ManagementSystems Moodle scheint es keine bedeutsamen Änderungen im Nutzungsverhalten 
zu geben. Einschränkend zu erwähnen ist jedoch, dass bereits zu MZP 1100 \% der Befragten angaben, Moodle zu nutzen. Zu beiden MZP lag die Abrufhäufigkeit tendenziell bei wöchentlich (siehe Abbildung 2 und Abbildung 3). Im Durchschnitt nutzen Studierende die Lernplattform fünf Mal pro Woche (MZP 2).

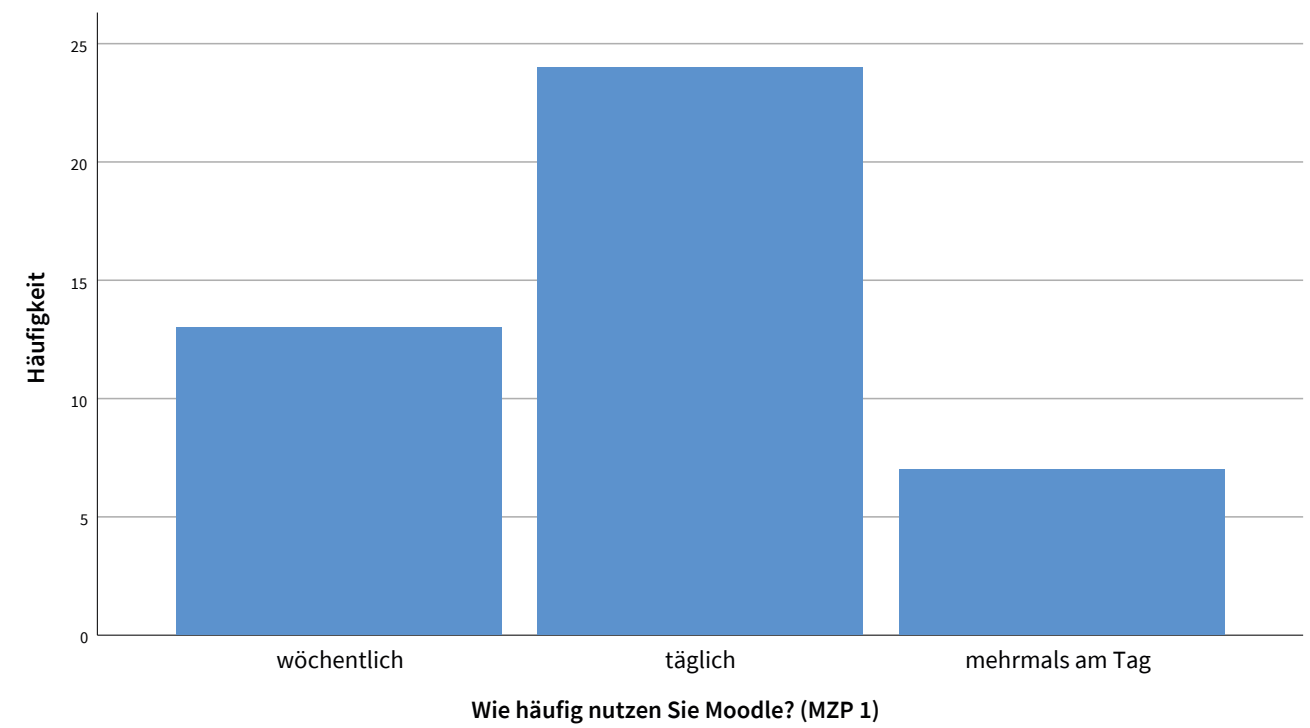

Abb. 2.: Nutzungshäufigkeit der Lernplattform Moodle MZP 1 (Eigene Darstellung).

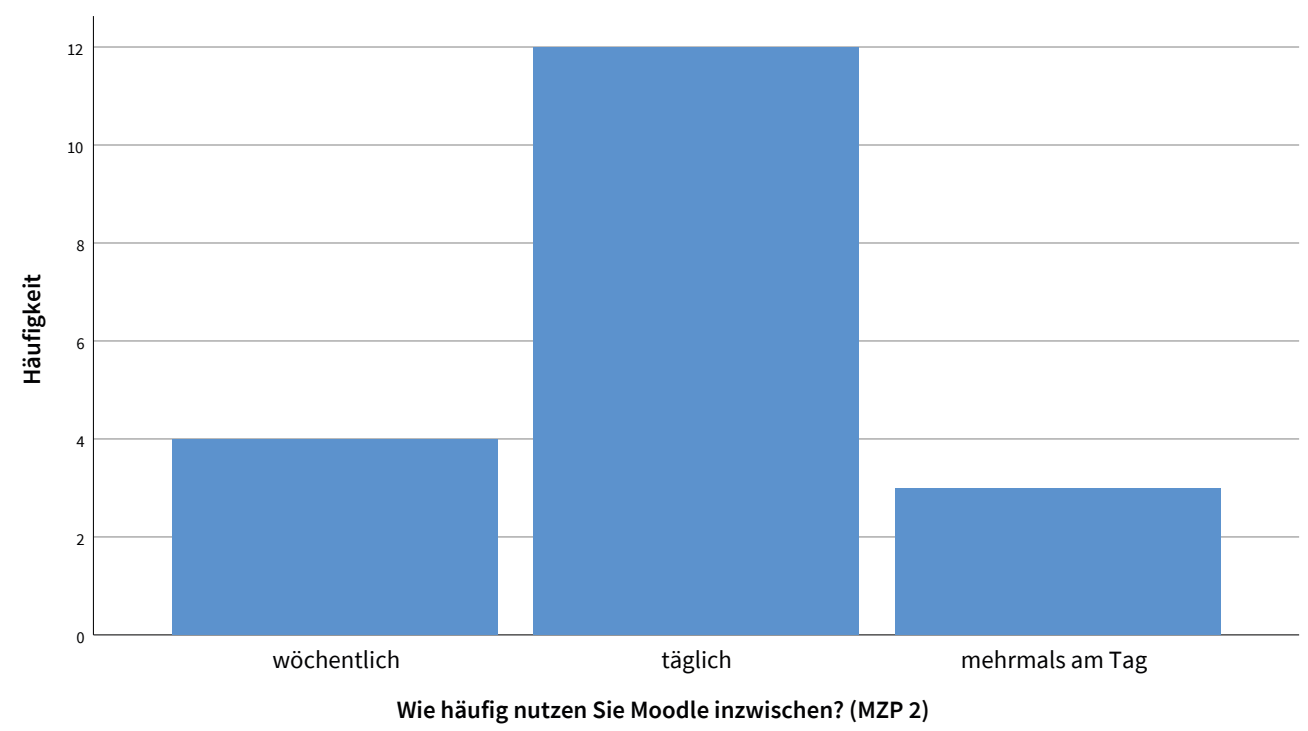

Abb. 3.: Nutzungshäufigkeit der Lernplattform Moodle MZP 2 (Eigene Darstellung). 


\subsection{Deskriptive Beschreibung genereller subjektiver studentischer Einstellungen zu digitaler Lehre}

Die Studierenden berichten in Freitextaussagen, sowohl synchrone als auch asynchrone Veranstaltungen erlebt zu haben. Es bestand die Annahme, Studierende würden asynchrone E-Learning Formate gegenüber synchronen bevorzugen. Während $16 \%$ synchrone digitale Lerneinheiten für effektiver halten, bevorzugen weitere $16 \%$ asynchrone Lerneinheiten und $68 \%$ halten beides für gleich effektiv. Es wurde vermutet, dass Studierende lieber zu definierten als zu flexibel setzbaren Zeiten lernen. Diesbezüglich berichten $37 \%$ lieber zu bestimmten von Lehrenden festgelegten Zeiten zu lernen, während $58 \%$ lieber zu individuellen Zeitpunkten, die selbst festgelegt werden, lernen.

$84 \%$ der Studierenden lernen lieber allein, während $16 \%$ angaben, lieber in Gruppen zu lernen («Gruppenarbeiten sind so schon eine schreckliche Sache für verantwortungsbewusste Studenten, weil man oft mit faulen Studenten arbeiten muss, was durch das digitale Zusammenarbeiten nochmal erschwert wurde.»). Das gilt allerdings, wie auch im Zitat deutlich wird, nicht ausschliesslich für digitale Lerneinheiten. Nichtsdestotrotz gibt es auch Studierende, die der Ansicht sind, dass das «Arbeiten in Kleingruppen gut funktioniert» hat.

Ob Studierende digitale oder analoge Elemente in Lehre und beim Lernen präferieren, scheint interindividuell verschieden zu sein. Während $16 \%$ es vorziehen würden, dass Studieren in Zukunft rein online möglich sein sollte, hoffen $21 \%$, dass Lehre wieder so stattfinden wird wie vor der Covid-19-Pandemie. Weitere $53 \%$ sind der Meinung, dass Lehrende in Zukunft mehr digitale Elemente in ihre Lehre integrieren sollen. Auf der anderen Seite befürworten weitere $10 \%$, dass der Anteil von analogen Elementen wieder zunehmen sollte. Auf die Frage, ob sie auch im nächsten Semester gerne digitale Lehrveranstaltungen belegen möchten, antworten $21 \%$ vollkommen, $26 \%$ überwiegend, $26 \%$ teilweise, $21 \%$ kaum und $5 \%$ gar nicht. Das Meinungsbild erscheint heterogen.

Trotz der kleinen Stichprobe wurden Zusammenhangsmasse zwischen Akzeptanz und Nutzen von E-Learning Angeboten und diversen Kontextfaktoren berechnet. Diese sind jedoch nur eingeschränkt aussagekräftig.

\subsection{Akzeptanz und Nutzungsverhalten}

Zwischen der Akzeptanz und der Nutzungshäufigkeit von E-Learning besteht ein signifikant positiver Zusammenhang $\left(r=.51, p=.03^{\star}\right)$. Studierende, die digitalen Lernangeboten positiv gegenüberstehen, nutzen derartige Formate häufiger und intensiver. 


\subsection{Sozioökonomische Faktoren}

Zwischen Parametern der sozioökonomischen Situation der befragten Studierenden und Akzeptanz und Nutzungsverhalten von E-Learning Formaten konnten kaum signifikante Zusammenhänge gefunden werden. Ob ein Voll- bzw. Teilzeitstudium, Elternschaft oder die Muttersprache mit Akzeptanz und Nutzen von digitalen Lehrund Lernangeboten zusammenhängen, konnte in dieser Untersuchung nicht getestet werden, da $100 \%$ in Vollzeit studieren, die Muttersprache bei nahezu allen Befragten deutsch ist und keine bzw. keiner der Befragten Kinder hat. Aber auch das Wohnen in einer Lebenspartnerschaft und die Distanz vom Wohnort zur Hochschule korrelieren nicht mit Akzeptanz und Nutzen von E-Learning Angeboten. Der Zugang zu stabilem Internet scheint dagegen eine entscheidende Rolle zu spielen. Wer über eine stabile Internetverbindung verfügt, akzeptiert eher E-Learning Formate $\left(r=.53, p=.02^{*}\right)$. Anzumerken ist an dieser Stelle, dass $68 \%$ der Befragten nur teilweise über eine stabile Internetverbindung verfügen.

\subsection{Organisatorische und didaktische Gestaltung}

Je besser die organisatorische Gestaltung eines E-Learning Angebots, desto höher deren Akzeptanz $\left(r=.54, p=.02^{\star}\right)$. Problemorientierte Unterstützung und das Ausbleiben technischer Schwierigkeiten hängen folglich mit erhöhter Akzeptanz zusammen. Wenn Unterstützungsbedarf bestand, empfanden $11 \%$ die Hilfe vollkommen, $42 \%$ überwiegend, $26 \%$ teilweise und $21 \%$ kaum als rasch und kompetent. Technische Probleme waren bei $42 \%$ kaum, weiteren $42 \%$ teilweise und $15 \%$ öfter vorhanden.

Auch die didaktische Gestaltung eines Angebots spielt eine Rolle für dessen Akzeptanz ( $r=.37, p=.12$ ), auch wenn dieser Befund knapp nicht signifikant ist. Je besser die dargebotenen Lerninhalte und Lehrkonzepte ausgearbeitet sind, desto höher gestaltet sich die Akzeptanz des digitalen Angebots. Insgesamt empfanden die Studierenden die didaktische Gestaltung wie folgt: $11 \%$ der Studierenden bewerteten die Lehr- und Lernkonzepte digitaler Lehr- und Lernangebote als vollkommen didaktisch gut aufbereitet und klar verständlich, $26 \%$ als überwiegend, $58 \%$ als teilweise und $5 \%$ als kaum. Übungen und Beispiele zur Festigung des Lehrstoffs waren für $21 \%$ vollkommen, $21 \%$ überwiegend, $37 \%$ teilweise und $21 \%$ kaum ausreichend vorhanden. Konstruktive Rückmeldungen durch Dozierende sind für $16 \%$ vollkommen, für $5 \%$ überwiegend, für $58 \%$ teilweise, für $16 \%$ kaum und $5 \%$ gar nicht genügend.

Zudem besteht zwischen organisatorischer und didaktischer Gestaltung selbst ein hochsignifikanter positiver Zusammenhang $\left(r=.78, p=.00^{\star \star \star}\right)$. Ist die organisatorische Gestaltung gelungen, ist die didaktische Gestaltung ebenso durchdacht und positiv bewertet. 


\subsection{Studien- und Lernmerkmale}

Die angenommenen Zusammenhänge zwischen Studienerfolg sowie Vorkenntnissen und Akzeptanz und Nutzen von E-Learning Formaten, liessen sich in dieser Untersuchung nicht bestätigen. Hinsichtlich ihres bisherigen Studienerfolgs, schätzen $32 \%$ ihren Erfolg als ausgezeichnet, $53 \%$ gut und $16 \%$ durchschnittlich ein. Auf die Frage, wie sie ihre potenzielle Studienleistung für das Sommersemester 2020 einschätzen, antworten die Studierenden wie folgt: $11 \%$ auf jeden Fall schlechter, $16 \%$ vermutlich schlechter, $37 \%$ eher nicht schlechter, $11 \%$ auf keinen Fall schlechter, und $26 \%$ kann ich nicht einschätzen. Beide Variablen korrelieren nicht signifikant mit Akzeptanz und Nutzen von E-Learning Formaten. Hinsichtlich ihrer Vorerfahrung mit digitalen Lernangeboten, auch ausserhalb der Universität, berichten $21 \%$ noch nie, $53 \%$ ein bis drei Mal und $26 \%$ öfter als drei Mal solche Formate genutzt zu haben. Inneruniversitär zeichnet sich ein ähnliches Bild ab. $21 \%$ haben noch nie, $68 \%$ ein bis drei Mal und $11 \%$ öfter als drei Mal mit E-Learning oder E-Learning-Elementen gearbeitet. Keine der beiden Variablen weist eine signifikante Korrelation zu Akzeptanz und Nutzen von E-Learning-Angeboten auf.

\section{Fazit und Blick in die Zukunft}

Hauptsächliches Ziel der vorliegenden Untersuchung war es, den derzeitigen Stand von Lehre und Lernen an einer deutschen Hochschule, geprägt von durch Covid-19 bedingten Veränderungen, zu skizzieren. Dabei wurden Bachelorstudierende der Disziplin Erziehungswissenschaften befragt. Die folgenden Interpretationen beziehen sich daher auf diese Subgruppe und müssen für weitere Gruppen erst verifiziert werden. Generell wurde eine sehr kleine Stichprobe dieser Subgruppe an einer einzigen Universität in Deutschland untersucht, sodass die aktuelle Studie lediglich einen ersten Eindruck der Hochschullehre während der Pandemie vermitteln will, jedoch keinen Anspruch auf allgemeine Gültigkeit erhebt.

Ernüchternd ist, dass Studieren nicht für alle Studierenden im Sommersemester 2020 problemlos möglich zu sein gewesen scheint. Ein bemerkenswert grosser Anteil verfügt nicht über einen Laptop bzw. PC oder hat keine stabile Internetverbindung. Ein ähnliches Bild zeichnet eine grösser angelegte Studie der Universität DuisburgEssen (Stammen und Ebert 2020). Ein Viertel der knapp 7.000 befragten Studierenden gibt an, nicht alle für das Online-Semester benötigten Geräte zu besitzen. Knapp $35 \%$ berichten die Notwendigkeit des Bedarfs einer Geräteanschaffung, um das Studium aufrechtzuerhalten. Chancengleichheit unter Studierenden kann somit während der Schliessung von Bibliotheken, PC-Pools usw. nicht garantiert werden. Hieraus ergibt sich gesellschaftspolitisch der unbedingte Bedarf der Schaffung von Anlaufstellen mit entsprechender Ausstattung, um allen Studierenden gleichermassen ein Studium in Regelstudienzeit zu ermöglichen. 
Zudem liefert die Studie Hinweise für heterogene Präferenzen und Bedürfnisse unter Studierenden hinsichtlich digitaler Lehre und Lernen. Zukünftige Studien sollten sich der Erforschung dieser Heterogenität widmen, um Chancengleichheit im Studium, auch in Zeiten, die ausschliesslich digitales Lernen und Lehren erfordern, zu ermöglichen.

Darüber hinaus scheint das Sommersemester 2020 aus Studierendenperspektive eine Herausforderung dargestellt zu haben und wird von einem Grossteil der in dieser Untersuchung aber auch in der Studie von Stammen und Ebert (2020) befragten Studierenden mit neuen Ansprüchen und Zeitaufwand assoziiert. Im Sinne der Cognitive Load Theory (u.a. Sweller 2011) entstand wohl für viele Studierende eine hohe kognitive Arbeitsbelastung, indem Lernprozesse durch fremde und teilweise lernunfreundliche Prozesse, Strukturen, Abläufe und Materialien erschwert wurden. Die extrinsische Belastung könnte in Folgesemestern beispielsweise durch die Optimierung von Lernmaterialien, indem überflüssige und irrelevante Informationen reduziert werden, oder durch klar strukturierte Prozessabläufe und verbesserte Kommunikationsstrukturen, verringert werden. Die Gewöhnung an digitale Lehr- und Lernkonzepte würde ebenso mit einer Reduktion extrinsischer Belastung einhergehen, was das studentische Lernen erleichtern könnte. Darüber hinaus scheint indiziert, dass Studierende in Zukunft besser auf digitale Lehr- und Lernszenarien vorbereitet werden sollten. Es braucht neben den einzelnen digitalen Kursen, übergeordnete Strukturen und konkrete Ansprechpersonen, die Studierenden sowohl bei inhaltlichen als auch bei organisatorischen Problemstellungen behilflich sind. Derartige Personen sollten in Zukunft auf mehreren Ebenen (Lehrstuhl, Fachbereich, Verwaltung usw.) angesiedelt sein und deren Zuständigkeitsbereiche klar definiert werden. Bedeutsam erscheint, wie generell niedrig die Studierenden ihre Kompetenzen im Umgang mit digitalen Medien einschätzen. Fraglich ist, ob derartige Kompetenzen tatsächlich so gering ausgeprägt sind oder nur als so wahrgenommen werden. Dennoch lässt sich ein Bedarf zur Förderung von Kompetenzen im Umgang mit digitalen Lehr- und Lernszenarien vermuten. Diese Vermutung wird bekräftigt durch den Befund, dass $21 \%$ angeben, noch nie und $68 \%$ erst ein bis drei Mal digitale Lehr- und Lernformate im universitären Kontext erlebt zu haben, obwohl sie sich bereits im vierten Fachsemester befinden.

Des Weiteren scheint den Studierenden Austausch mit anderen Studierenden und Dozierenden zu fehlen. Kommunikations- und Kooperationsanlässe zu schaffen, ist demnach im Sommersemester 2020 mutmasslich nicht gänzlich gelungen. In Folgesemestern, die ausschliesslich digitales Lernen erfordern könnten oder in denen digitale Elemente im Lehrangebot einen höheren Anteil einnehmen, sind Lehrende dazu aufgefordert, mehr Anstösse zur Diskussion und Zusammenarbeit zu schaffen. Diesbezüglich müssten auch Lehrende gefördert werden, indem ihnen einerseits geeignete digitale Methoden und Werkzeuge nahegebracht werden, andererseits 
Ängste und Vorbehalte genommen und Partizipations- und Innovationsbereitschaft gesteigert werden (Kriegesmann und Kley 2012). Studierenden scheint eine solche Gewöhnung an fremde digitale Formate gut zu gelingen. Ein Grossteil der befragten Studierenden hat sich im Sommersemester 2020 mit neuen digitalen Werkzeugen auseinandergesetzt, hat erste Hürden überwunden und kann somit die gewonnenen Kompetenzen im Umgang mit digitalen Werkzeugen und Artefakten auch in Zukunft zu Lernzwecken nutzen (auch Stammen und Ebert 2020). Die Annahme jedoch, dass die digitale Affinität der Studierenden im Verlauf des Sommersemesters 2020, also während der Pandemie, zugenommen hat, konnte in dieser Untersuchung nicht ermittelt werden. Folgestudien könnten dies in grösseren Stichproben und über die gesamte Dauer der Pandemie prüfen.

Die durchgeführte Studie liefert auch Hinweise zu generellen studentischen Präferenzen bezüglich digitaler Lehr- und Lernformate. Die Präferenzen hinsichtlich Synchronität, Selbststrukturierung, Kooperation sowie digitalen und analogen Anteilen scheinen jedoch interindividuell stark zu variieren. Interindividuelle Unterschiede im Lernverhalten zwischen Studierenden sind vielseits bekannt (Persike und Friedrich 2016; Metzner et al. 2019). Die Covid-19-Pandemie zwang Lehrende jedoch oftmals zu einer ad hoc Digitalisierung ihrer Lehre. Der Anspruch, der breiten Masse der Studierenden dieselben Lerninhalte zu vermitteln und dieselben Lehr- und Lernziele zu erreichen wie in den Semestern zuvor, stand für Lehrende wohl meist im Fokus. Einzelne Schwierigkeiten seitens der Studierenden rückten hingegen in den Hintergrund. In Zukunft sollten interindividuell verschiedene Anforderungen und Bedürfnisse identifiziert und angemessene Massnahmen (z. B. Schaffung von zeitlich stabilen studierendeninternen Videokonferenzräumen, virtuelle und terminierte Sprechstunden durch Lehrende und Tutorinnen bzw. Tutoren) etabliert werden. Weitere Forschung könnte darüber hinaus untersuchen, von welchen Persönlichkeitsaber auch Umgebungsvariablen es abhängt, wie gut das digitale Studieren funktioniert. Eine gross angelegte Studie von Persike und Friedrich (2016) zeigte bereits, dass die Nutzung digitaler Medien zwischen Studierenden stark variiert, abhängig von Studienfach- und Hochschulzugehörigkeit.

Ein weiteres Ziel der vorliegenden Untersuchung bestand in der Analyse von Faktoren, die Akzeptanz sowie Nutzung von E-Learning Angeboten durch Studierende beeinflussen. Wie in der Studie von Kreidl (2011), zeigten sich auch in dieser Untersuchung kaum Zusammenhänge zwischen sozioökonomischen Faktoren sowie Studien- und Lernmerkmalen mit Akzeptanz und Nutzung von digitalen Lehr- und Lernformaten. Lediglich der Zugang zu stabilem Internet korrelierte in dieser Studie positiv mit der Akzeptanz der Studierenden. Zwischen Akzeptanz und organisatorischen sowie didaktischen Faktoren liessen sich bedeutsame Zusammenhänge nachweisen. Je besser das didaktische und organisatorische Konzept ausgearbeitet ist, desto höher die Akzeptanz für den digitalen Kurs seitens der Studierenden. Diese 
Feststellung, auch wenn sie auf einer kleinen Untersuchung beruht, sollte Lehrende darin bekräftigen, organisatorische Rahmenbedingungen zu berücksichtigen und mediendidaktische Konzepte zu integrieren.

Insgesamt weisen die Daten dieser Studie daraufhin, dass die didaktische Konzeption von Lehre und Lernen ausbaufähig ist. Ein Grossteil der befragten Studierenden empfindet Aufbereitung und Verständlichkeit der dargebotenen Lerninhalte und -konzepte, Gelegenheiten zum Üben und Beispiele sowie konstruktives Feedback nur teilweise ausreichend. Korrelationen mit der Nutzung von E-Learning Formaten wurden in der vorliegenden Untersuchung nicht gefunden. Allerdings wurde dieses Konstrukt recht kurz und lediglich subjektiv erfragt. Für zukünftige Studien wäre es wünschenswert, Lernprozesse und Lernerfolge nicht ausschliesslich über Akzeptanzund Nutzungsparameter zu erfragen, sondern komplexere multidimensionale Konstrukte, wie Student Engagement (u. a. Kahu 2013; Bedenlier et al. 2020), das affektive, kognitive und behaviorale Komponenten integriert, heranzuziehen. Auch LearningAnalytics-Daten (z. B. Abrufhäufigkeit von Moodle, die Anwesenheitszeit oder Abbruchquote bei einer synchronen Online-Veranstaltung) könnten validere Ergebnisse als Selbstauskunftsverfahren liefern. Die vorliegende Arbeit kann den Standards wissenschaftlichen Arbeitens teilweise nicht gerecht werden. Es wäre wünschenswert, dass zukünftige Studien grössere Stichproben untersuchen, auch in longitudinalen Settings. Auffällig in dieser Untersuchung ist ausserdem, dass $100 \%$ der Befragten in Vollzeit studieren. Es ist anzunehmen, dass die Daten der Stichprobe nicht repräsentativ sind (Metzner et al. 2019). Nichtsdestotrotz liefert die Studie erste wichtige Hinweise zu studentischen Präferenzen und Einstellungen hinsichtlich digitaler Lehre. Es ist dennoch zu vermuten, dass die Untersuchung anderer Stichproben andere Ergebnisse geliefert hätte. So hat sich bereits gezeigt, dass bestimmter Lehrstoff eher für die Vermittlung über Online-Lehre geeignet ist als anderer. Insbesondere bei computeraffinen Disziplinen wie Informatik oder Mathematik sowie bei Fächern mit hohen Studierendenzahlen eignen sich virtuelle Lehrveranstaltungen (Persike und Friedrich 2016; Schmid, Thom und Görtz 2016; Metzner et al. 2019).

Die Ergebnisse der vorliegenden Studie gehen einher mit den Modellen und theoretischen Ausführungen von Kerres (2018), Euler und Seufert (2005) sowie Kreidl (2011). Organisatorische, technologische, aber auch didaktische Kriterien erscheinen, wie die vorliegende Studie aus Studentenperspektive aufzeigt, von grosser Relevanz für eine erfolgreiche und nachhaltige Verankerung von digitalem Lernen in der Hochschullehre und werden von den Studierenden teilweise als ausbaufähig wahrgenommen. Daraus leitet sich die Handlungsempfehlung für Hochschulen als Organisationen sowie für Lehrende als Individuen ab, bei der Konzeption digitaler Lehre besonders auf didaktische und organisatorische Gestaltungsaspekte zu achten. Lehrenden ist das Werk von Handke (2020) zu empfehlen, der getrieben durch die Herausforderungen der Pandemie Umsetzungsideen für digitale Hochschullehre 
entwickelte und anhand von Beispielen Wege aufzeichnete, wie der Einstieg in die Digitalisierung gelingen kann. Aber auch Studierende scheinen nicht ausreichend vorbereitet für das Online-Semester 2020 gewesen zu sein. Es hat den Studierenden zumindest aus subjektiver Sicht wahrscheinlich an Kompetenzen hinsichtlich digitalen Lernens gemangelt. Daraus ergibt sich die unbedingte Notwendigkeit der Schaffung optionaler Support-Instanzen an Hochschulen, die Studierende abhängig von ihren Vorkenntnissen und Bedürfnissen auf digitale Lehre vorbereiten und fortlaufend unterstützen.

Die digitale Transformation der Hochschullehre im Speziellen und des Hochschulsektors im Allgemeinen erfordert die aktive Mitarbeit und Innovationsbereitschaft verschiedener Akteurinnen und Akteure. Nur durch das Engagement und die Zusammenarbeit von Studierenden, Lehrenden und dem Verwaltungsapparat kann der Veränderungsprozess, der durch die Covid-19-Pandemie an Fahrt aufgenommen hat, langfristig gelingen.

\section{Literatur}

Becker, Michael, Tim Riplinger, und Mandy Schiefner-Rohs. 2020. «Von Hoffnungen und Enttäuschungen gegenseitiger Erwartungen an Lehre». In Studierende-Medien-Universität. Einblicke in studentische Medienwelten, herausgegeben von Sandra Hofhues, Mandy Schiefner-Rohs, Sandra Aßmann, und Taiga Brahm. 87-105. Münster: Waxmann. https:// doi.org/10.31244/9783830990499.

Bedenlier, Svenja, Melissa Bond, Katja Buntins, Olaf Zawacki-Richter, und Michael Kerres. 2020. «Facilitating student engagement through educational technology in higher education: A systematic review in the field of arts and humanities.» Australasian Journal of Educational Technology, 126-50. https://doi.org/10.14742/ajet.5477.

Bremer, Claudia, Marc Göcks, Paul Rühl, und Jörg Stratmann. 2010. «Einleitung.»In Landesinitiativen für E-Learning an deutschen Hochschulen, herausgegeben von Claudia Bremer, Marc Göcks, Paul Rühl und Jörg Stratmann, 7-12. Medien in der Wissenschaft 57. Münster: Waxmann.

Ehlers, Ulf-Daniel. 2011. Qualität im E-Learning aus Lernersicht. 2. Aufl. Medienbildung und Gesellschaft 15. Wiesbaden: VS Verlag für Sozialwissenschaften. https://doi.org/10.1007/9783-531-93070-1.

Euler, Dieter, und Sabine Seufert. 2003. Nachhaltigkeit von eLearning-Innovationen: SCIL-Arbeitsbericht 1. St. Gallen. https://www.scil.ch/produkt/arbeitsbericht-1-nachhaltigkeitvon-elearning-innovationen/.

Euler, Dieter, und Sabine Seufert. 2005. «Von der Pionierphase zur nachhaltigen Implementierung - Facetten und Zusammenhänge einer pädagogischen Innovation.» In E-Learning in Hochschulen und Bildungszentren, herausgegeben von Dieter Euler und Sabine Seufert, 1-24. E-Learning in Wissenschaft und Praxis 1. München: De Gruyter Oldenbourg. 
Faber, Konrad. 2011. «Einleitung.» In Vernetzung schafft Perspektive: Neue Ansätze in der Lehrerbildung, herausgegeben von Rolf Arnold und Konrad Faber. 1. Aufl., 9-13. Grundlagen der Berufs- und Erwachsenenbildung 67. Baltmannsweiler: Schneider Verlag Hohengren.

Handke, Jürgen. 2020. Handbuch Hochschullehre Digital: Leitfaden für eine moderne und mediengerechte Lehre. 3. Aufl. Marburg: Tectum Wissenschaftsverlag.

Kahu, Ella R. 2013. «Framing Student Engagement in Higher Education.» Studies in Higher Education 38 (5): 758-73. https://doi.org/10.1080/03075079.2011.598505.

Kergel, David, und Birte Heidkamp-Kergel. 2020. E-Learning, E-Didaktik und digitales Lernen. Wiesbaden: VS Verlag für Sozialwissenschaften. https://doi.org/10.1007/978-3-658-282776.

Kerres, Michael. 2018. Mediendidaktik: Konzeption und Entwicklung digitaler Lernangebote. 5. Aufl. Berlin: De Gruyter Oldenbourg. https://doi.org/10.1515/9783110456837.

Kerres, Michael. 2020. «Against All Odds: Education in Germany Coping with Covid-19. Postdigital Science and Education.» Postdigital Science and Education. https://doi.org/10.1007/ s42438-020-00130-7.

Kleimann, Bernd, und Klaus Wannemacher. 2005. «E-Learning-Geschäftsmodelle für Hochschulen.»In E-Learning: Einsatzkonzepte und Geschäftsmodelle, herausgegeben von Michael H. Breitner und Gabriela Hoppe, 225-40. Heidelberg: Physica-Verlag.

Klinger, Sabine. 2015. "Das ,feminisierte‘ Studium der Erziehungs- und Bildungswissenschaften und die studentische (De-)Thematisierung von Geschlecht und Geschlechterfragen.» Gender: Zeitschrift für Geschlecht, Kultur und Gesellschaft 7 (1): 113-28. https://doi. $\operatorname{org} / 10.25595 / 41$.

Kreidl, Christian. 2011. Akzeptanz und Nutzung von E-Learning-Elementen an Hochschulen: Gründe für die Einführung und Kriterien der Anwendung von E-Learning. Medien in der Wissenschaft 59. Münster: Waxmann.

Kriegesmann, Bernd, und Thomas Kley. 2012. Mitbestimmung als Innovationstreiber. 1. Aufl. Forschung aus der Hans-Böckler-Stiftung 141. Baden-Baden: edition sigma.

Lehmann, Robert. 2010. Lernstile als Grundlage adaptiver Lernsysteme in der Softwareschulung (Medien in der Wissenschaft, Bd. 54). Münster [u.a.]: Waxmann.

Lojewski, Johanna, und Miriam Schäfer. 2017. «Digitale Ungleichheit - in der Hochschule?». In Gestaltung des Sozial- und Gesundheitswesens im Zeitalter von Digitalisierung und technischer Assistenz, herausgegeben von Tim Hagemann, 419-38. Forschung und Entwicklung in der Sozialwirtschaft 11. Baden-Baden: Nomos.

Metzner, Joachim, Ulrich Bartosch, Melanie Vogel, Anja-Lisa Schroll, Martin Rademacher, und Hubertus Neuhausen. 2019. Was bedeutet Hochschullehre im digitalen Zeitalter? Eine Betrachtung des Bildungsbegriffs vor den Herausforderungen der Digitalisisierung. Arbeitspapier 50. Berlin. https://hochschulforumdigitalisierung.de/sites/default/files/dateien/ HFD_AP_Nr50_Hochschullehre_im_digitalen_Zeitalter_web.pdf. 
Persike, Malte, und Julius-David Friedrich. 2016. Lernen mit digitalen Medien aus Studierendenperspektive: Sonderauswertung aus dem CHE Hochschulranking für die deutschen Hochschulen. Arbeitspapier. Berlin. https://hochschulforumdigitalisierung.de/sites/default/ files/dateien/HFD_AP_Nr_17_Lernen_mit_digitalen_Medien_aus_Studierendenperspektive.pdf.

Rüggeberg, Harald. 2009. Innovationswiderstände bei der Akzeptanz hochgradiger Innovationen aus kleinen und mittleren Unternehmen. Business \& Management Working Papers (51), 1-31. http://www.mbaberlin.de/fileadmin/user_upload/MAINdateien/1_IMB/Working_Papers/2009/WP51_Rueggeberg_12-2009.pdf

Schmid, Ulrich, Sabrina Thom, und Lutz Görtz. 2016. Ein Leben lang digital lernen: Neue Weiterbildungsangebote aus Hochschulen. Arbeitspapier 20. Berlin: Hochschulforum Digitalisierung. Zugriff am 21. August 2020. https://hochschulforumdigitalisierung.de/sites/default/ files/dateien/HFD_AP_Nr20_Lebenslanges_Lernen.pdf.

Schünemann, Isabel, und Jannica Budde. 2018. Hochschulstrategien für die Lehre im digitalen Zeitalter. Arbeitspapier 38. Berlin: Hochschulforum Digitalisierung. Zugriff am 21. August 2020. https://hochschulforumdigitalisierung.de/sites/default/files/dateien/HFD_AP_ Nr38\%20Empfehlungen_Strategieentwicklung_WEB.pdf.

Stammen, Karl-Heinz, und Anna Ebert. 2020. «Noch online? Studierendenbefragung zur medientechnischen Ausstattung im Sommersemester 2020.» Zugriff am 21. August 2020. https://panel.uni-due.de/assets_websites/18/StammenEbert_2020_NochOnline_Gesamtbericht.pdf.

Sweller, John. 2011. «Cognitive Load Theory.»In The Psychology of Learning and Motivation, herausgegeben von Jose P. Mestre und Brian H. Ross. Psychology of Learning and Motivation 55. Cambridge: Academic Press.

Wannemacher, Klaus, Imke Jungermann, Sven Osterfeld, Julia Scholz und Anna von Villiez. 2016. Organisation digitaler Lehre in den deutschen Hochschulen. Hochschulforum Digitalisierung beim Stifterverband für die Deutsche Wissenschaft eV. 